\title{
NOTE SUR LA MÉTHODE D'ÉLEVAGE DE L'ALEVIN DE CARPE EY FRAYÈRES DLBISCH“ ET BASSIN DE GROSSISSEMENT
}

\author{
par P. CHIMITS \\ Ingenjeur agronome, Inspecteur des Eaux et Forêts \\ et S. KRECZMER \\ Ingénieur agronome S.G.G.W. de l'Universite de Varsopie
}

(Fin) (1)

VII. - Résultats deg expentences faites a Counville en i946

$1^{\circ}$ Bassins de ponte. - Deux bassins de ponte ont été créés.

Le $28 \mathrm{Mai}$, une femelle de 8 kilos et deux mâles de 5 kilos et 4 kilos ont été mis en place. La ponte retardée par un orage dans la nuit du $28 \mathrm{Mai}$ au 29 Mai a eu lieu le $3 o$ Mai au matin et l'éclosion le 3 Juin.

La pêche des alevins a été effectuée le 6 Juin.

Dans une nouvelle expérience de ponte, pour permettre une prise de vue cinématographique, la mise en place des géniteurs a eu lieu le 8 Juin, la ponte le 9 Juin. Les alevins éclos à cètte deuxième ponte n'ont pas été utillisés.

$2^{\circ}$ Bassin de grossissement. - Le bassin de grossissement a une surface de $200 \mathrm{~m}^{2}$ et a êté préparé comme indiqué plus haut, la mise en eau a eu lieu le 30 Mai et la mise en place des alevins le 6 Juin, à raison de 150 au $\mathrm{m}^{3}$, soit 30.000 au total; les alevins restrnt dans le bassin de ponte ont été détruits, sauf un certain nombre qui ont été placés dans les étangs $4,5,6$ et $\mathrm{Ir}$.

Le plancton est abondant et se présente sous forme de véritables nuages de daphnies.

Ci-dessous l'anallyse du plancton faite par la Station Centrale d'Hydro. biologie appliquée.

Le volume liquide filtré par le filet à plancton (d'ailleurg à mailles relativement larges) était de $94.200 \mathrm{~cm}^{3}$ (filet de o m. 20 de diamètre traîné sur 3 mètres).

Le zooplancton est excessivement pauvre en espèces; il se compose

(1) Voir Bulletin : - no 142, Juillet-Septembre 1946, p. 30. 
presque exclusivement de Daphnia longispina, var. hyalina. Voici d'ailleurs I'abondance :

8 Juin. - Volume de plancton après sédimentation $: 5,5 \mathrm{~cm}^{3}$. Daphnia longispina var, hyalina.

15 Juin. - Volume du plancton après sédimentation : $14 \mathrm{~cm}^{3}$. Daphnia longispina dominant.

Larves de Corethra plumicornis = Chaoburus; quelques spécimens.

Volvox.

Spirogyres et Algues filamenteuses très abondants.

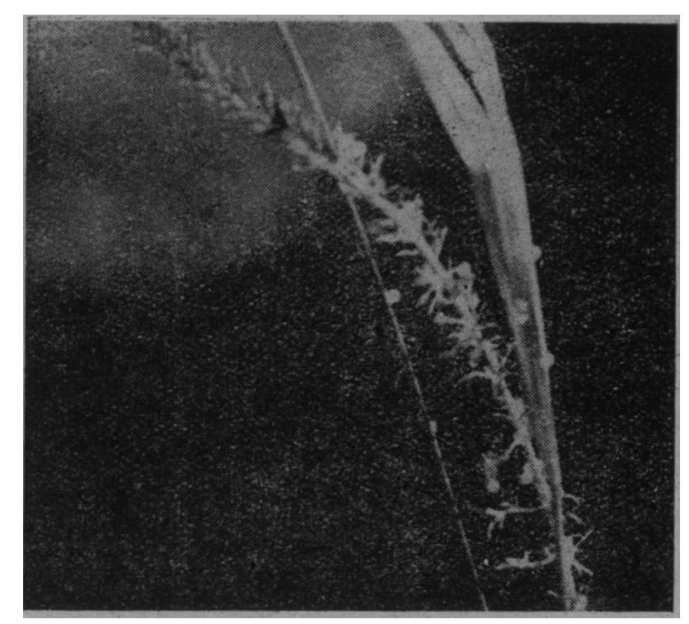

Fic. 20. - Brin d'herbes du plateau de fraye portant des ceuts de Carpes.

28 Juin. - Trop pauve pour etre mesuré.

Daphnia longsipina ; spécimens.

Volvox; quelques spécimens.

Insectes (débris).

Spirogyres et Algues filamenteuses, assez abondants.

Débris végétaux abondants.

On remarquera que le plancton qui, le 8 Juin, est d'abondance moyenne $\left(5,5 \mathrm{~cm}^{3}\right.$ à l'unité) au moment de la mise en place des petits alevins, continue à augmenter en quantité pendant huit jours ( $/ 4 \mathrm{~cm}^{3}$ le $15 \mathrm{Juin}$ ), puis diminue lentement pour devenir rare le 28 Juin. Il y a lieu de noter que le temps en Juin, pluvieux, peu chaud et sans soleil, a gêné fortement le développement du plancton; mais il faut surtout tenir compte de la variation relative de l'abondance du plancton. 
Le 28 Juin, il a été sorti 25.000 alevins mesurant de 20 à 35 millimètres, particulièrement gras, bien nourris et rigoureux.

On remarquera, que dans cette expérience, il a été mis dans les bassinns de grossissement i jo allevins au $\mathrm{m}^{2}$, au lieu de 50 comme nous le recommandons plus haut, afin d'indiquer les bons résultats que peuvent donner la méthodc. Il est bien évident qu'il est plus prudent de mettre moins de 150 alevins au $\mathrm{m}^{2}$ si on veut les tenir plus de quatre semaines et les obtenir plus gros.

$3^{\circ}$ Etang d'un été. - L'étang choisi a été l'étang 7 de l'établissement domanial de Courville. Il a une superficie de $I$ hectare 7 , une profondeur faible ( 1 mètre environ en moyenne), et contient une végétation immergée abondante. D'après les résultats obtenus les annnées"précédentes, c'est un des moins riches des huit étangs de l'établissement.

Toutefois, cet étang avait été soigneusement préparé. Chaulé à l'automne, laissé à sec tout l'hiver et le printemps, il a été faucardé à sec juste avant la mise en eau au début de Mai et a reçu alors une forte dose de scories (200 kilos en tout, soit environ 120 kilos à l'hectare). Nous escomptions pouvoir mettre du fumier en Juillet, comme indiqufí précédemment. Sur ces données, nous avons estimé à 270 kilos à l'hectare la productivité de notre étang.

Nous désirions obtenir à l'automne de la feuille de 30 grammes. Ceci nous donnait, comme quantité d'alevirıs à récolter à l'hectare :

270 kilos : o k. $03=9.000$ alevins, soit 15.300 alevins pour la surface totale de Il'étang.

Nous avons, en conséquence, déversé un nombre d'alevins supérieur de $30 \%$, pour tenir compte des pertes, soit 19.990 ou 20.000 en chiffres ronds.

Ces alevins ont été mis en place le 28 Juin.

Malheureusement, il nous fut, a cette époque, impossible d'obtenir du fumier, et nous eûmes quelques craintes d'avoir un peu trop surestimé la productivité et déversé trop d'alevins.

Pendant tout l'été, nous ne vîmes que rarement des alevins dans l'étang, ce qui, d'ailleurs, est bon signe, et prouve que la Carpe trouve sa nourriture et $n$ 'est pas malade.

$4^{\circ}$ Résultats de la pêche de l'étant d'un êté. - L'étang fut pêché les 23 et 24 Octobre 1946 , soit moins de quatre mois après l'immersion des alevins. Il fut récolté 16.265 alevins pesant au total 460 kilos 900 , d'une taille et d'une forme remarquablement homogènes, comme le fait ressortir le décompte ci-dessous :

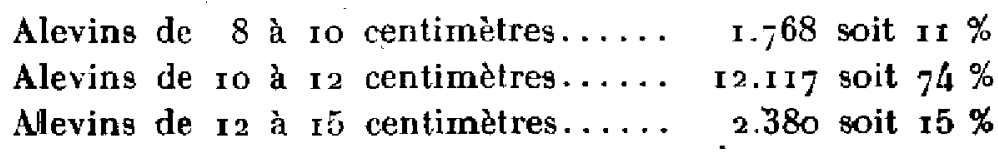


Le poids moyen de l'alevin ressort à $2 \delta$ gr. 3 et la productivité de l'étang à 271 kilos:

Nos calculs étaient donc remarquablement oonfirmés puisque nous obtenions un poids moyen à peine inférieur aux 30 grammes escomptés, et un chiffre d'alevins à peine supérieur (environ $7 \%$ ) au nombre escompté.

Nous devons toutefois reconnaître que nous avions estimé la productivité à $27^{\circ}$ kilos en tenant compte des épandages estivaux de fumier qui n'ont pu être exécutés. Nous avions donc, en réalité, sous-estimé la productivité que nous pourrons donc, à l'avenir, chiffrer à 300 ou 320 kilos pour l'étang 7 , préparé comme nous l'avons indiqué plus haut.

Quant à la productivité de l'étang 4 de Courville, elle pourrait, dans ces conditions, approcher de 400 kilos a l'hectare.

\section{- Vili. - Conbidérations phatiQues}

Ces chiffres peuvent, à première vue, paraitre forts, puisqu'on admet qu'en France le rendement moyen des étangs est de 100 kilos à l'hectare seulement, chiffre porté à 200 ou 250 kilos pour des étangs bien entretenus et fumés ; les chiffres de 300 kilos et au-dessus étant admis seulement pour les étangs exceptionnels ou pour ceux qui reçoivent de la nourriture artificielle.

Nous croyons que l'on peut arriver à ce chiffre de 300 kilos par des fumures rationnelles et sans nourriture artificielle.

Quant au rendement en argent de l'opération, si nous estimons it I50 francs le kilo d'alevins de Carpe de 30 grammes (ce qui est un prix plutốt faible), l'étang 7 aura donné, en 1946, un revenu brut d'envirou 40.000 francs à l'hectare.

Les frais d'exploitation (gardiennage, faucardage, engrais, pêche) atteignent environ $\mathbf{5} 5.000$ francs ì l'hectare.

\section{IX. - Quelques résultats de La méthode mixte employée DaNS Les ÉTANGg voIBINg}

Dans d autres étangs de Courville (étangs $4,5,6$ et il) nous avons déversé quelques dizaines de milliers d'alevins de quatre jours, sortant du bassin de ponte, et environ 6.000 alevins de un mois sortant du bassin de grossissement.

En outre, dans certains de ces étangs ( 4 et $1 \mathrm{I}$ ), nous avions mis un couple de géniteurs Carpe, en plus de géniteurs Tanche (i a à l'hectare environ) et de géniteurs Gardon (40 à l'hectare).

Les résultats de la pêche en alevins de Carpe (les alevins de Tanche et Gardon ayant été produits assez abondamment dans ceś étangs) sont consignés dans le tableau ci-dessous : 


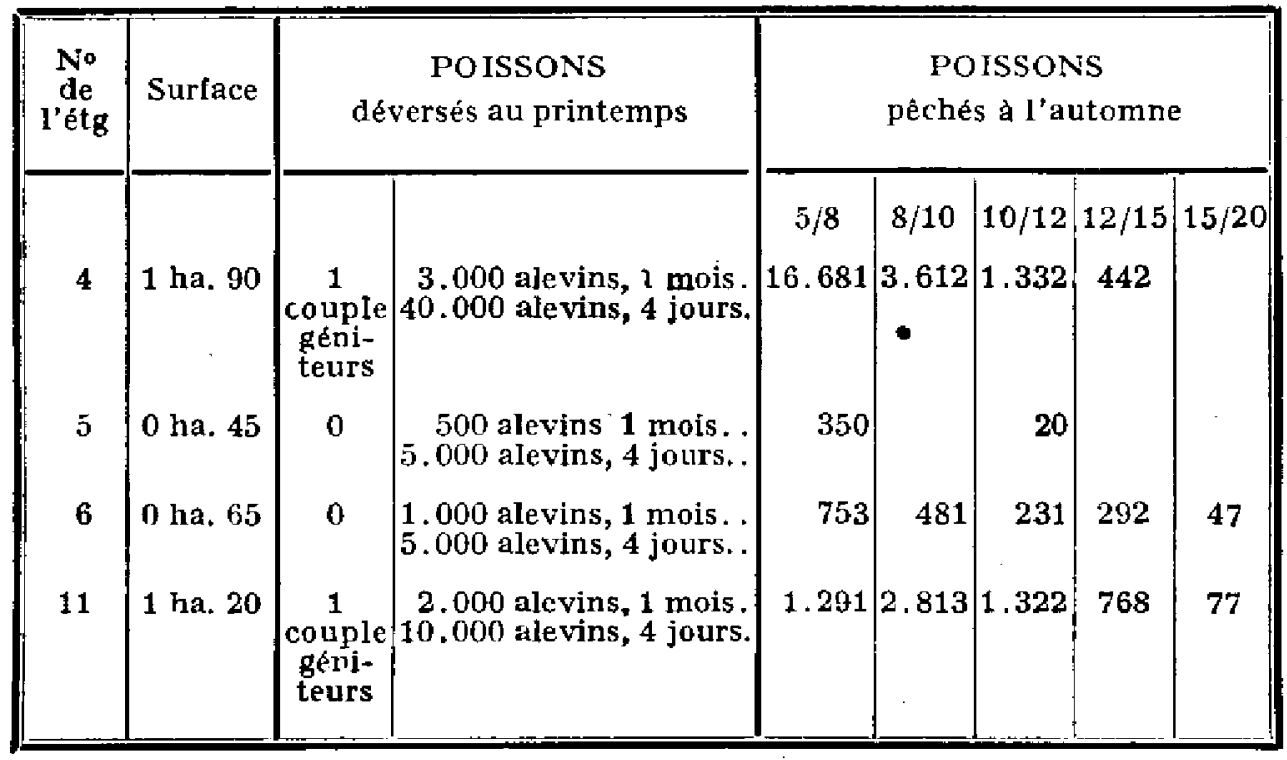

N. B. - La grille de séparation entre l'étang 5 et l'étang 4 ayant été détériorée, il y a lieu de supposer qu à la pèche tout au mołn", quelques alevins sont passées dans l'étang 4 .

Exception faite de l'étang 4 qui a obtenu un nombre important d'alevins $5 / 8$, les résultats ci-dessus montrent d'une manière frappante qu'en dehors d'un meilleur rendement, en nombre et en poids, les alevins obtenus par lạ méthode Dubisch pure, sont d'une taille beaucoup plus homogène.

Ils se répartissent, en effet, en trois catégories de vente : 8/1o centimètres, ro/ 12 centimètres, $12 / 15$ centimètres, avec prédominance de $74 \%$ dans la catégorie moyenne ro/ 22 centimètres, allors que dans tous les autres étangs ils s'étalent sur cinq catégories, de $5 / 8$ à $15 / 20$, sans prédominance de la catégorie moyenne.

Nous avons pu constater que dans les étangs 4 et ir, la fraie naturelle avait eu lieu normalement. Quand nous avons déversé nos alevins de quatre jours, nous avons constaté la présence, dans ces deux étangs; d'alevins de Carpe provenant de la fraie naturelle et déjà assez gros. Il est naturel de penser que la majorité des aleving $5 / 8$ proviennent de nos alevins de quatre jours.

\section{X. - NEGEgsite de RÉduIne AU MINIMUM tes REPRODUCTEURB dans la méthode de L'Étang de pose}

Dans les étangs de Courville où nous avons employé, pour produire nos alevins de Carpe, la méthode de l'étang de pose dont les résultats ont été donnés ci-dessus, nous aviọns, au printemps, réduit le nombre des géni- 
teurs Carpes au minimum, c'est-à-dire un couple de Carpes à l'hectare (géniteurs de 5 à 6 kilos en moyenne).

Les deux étangs de pose ainsi garnis, ont donné, pour une surface totale de 3 hectares ro, un résultat total de 28.000 alevins, soit environ 9.000 alevins à l'hectare, dont, il est vrai, une majorité d'alevins $5 / 8$.

Il convient de noter qu'outre la reproduction de ces géniteurs, il a été déversé dans ces étangs 5.000 alevins de un mois et 60.000 allevins de quatre jours.

Les années précédentes, on mettait en place plusieurs couples de reproducteurs à 1 'hectare, en moyenne 4 à 5 couples.

Or, la production d'alevins était bien inférieure et s'élevait, pour les six étangs du domaine, d'une surface d'environ 6 hectares et recevant de 60 à 80 géniteurs Carpes (outre les géniteurs Tanches et Gardong) à :

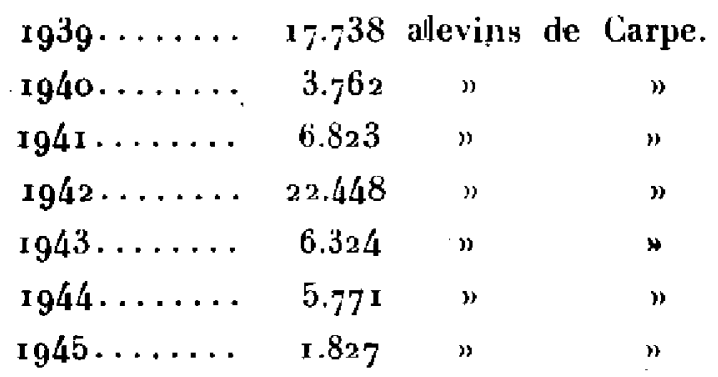

La moyenne de ces sept années fait donc ressortir une production annuelle d'environ r.50o alevins de Carpe par hectare et par an.

La moyenne des deux étangs 4 et I I donne, pour l'annnée I946, avec un seul couple de reproducteurs par étang, une production de 9.00o alevins à l'hectare.

Il n'est pas tenu compte de la production très importante des alevins de Tanches et Gardons. L'expérience dẽ cette année nous conduit à penser que plus le nombre de reprơducteurs Carpes dans un étang de pose est réduit, et plus les alevins sont abondants.

Nous estimons que dans un étang de pose de i à 4 hectares, un couple suffit amplement. Comme il n'est pas prudent de $n^{\top} \mathrm{en}$ mettre qu'un en raisỏn des Loutres, il est préférable de mettre deux femelles et trois máles.

Nous proposons, en mème temps, pour les pisciculteurs que l'emploi immédiat et sans experience préalable de la méthode des frayères et du bassin de grossissement pourrait effrayer, la méthode mixte décrite cidessus et consistant en l'utilisation tardive de simples frayères Dubisch sans bassin de grossissement, avec déversement d'alevins de quatre jours dans un étang de pose ne contenant qu'un couple de géniteurs ou deux femelles et trois måles. 


\section{XI. - Conclusions}

Des expériences que nous avons poursuivies à Courville en r946, nous pensons pouvoir déduire que :

$x^{\circ}$ La meilleure méthode d'obtention d'alevins de bonne race, calibrés, en quantité et taille désirable et calculable, est la méthode d'élevage en frayères Dubisch et bassin de grossissement.

$2^{\circ}$ La méthode mixte du bassin de ponte et de l'étang de pose peut donner de bons résultats si l'on réduit les gréniteurs à un couple ou par mesure de sécurité, à deux femelles et trois mâles, même si l'étang de pose a quelques hectares.

(Tous droits de reproduction réservés).

NOTA. - Si cette note suscite de l'intérêt auprès des pisciculteurs d'étangs, nous sommes disposés à leur donner tous renseignements utiles, et, au besoin, à organiser un petit stage d'application de quelques jours au Der et à Courville, en Juin 1947. Ln tel stage, sous l'égide de l'Administration des Eaux et Forêts et de la Station Centrale d'Hydrobiologie appliquée, permettrait, outre la pratique de la méthode, de donner des instructions sur la mesure du PH, l'hydrotimétrie, l'épandage les engrais, l'étude quantitative du plancton, etc... 